\title{
Compositional Decoupling of Bulk and Surface in Open-Structured Complex Mixed Oxides
}

\author{
Liudmyla Masliuk, Franz-Philipp Schmidt, Walid Hetaba, Milivoj Plodinec, Gudrun Auffermann, \\ Klaus Hermann, Detre Teschner, Frank Girgsdies, Annette Trunschke, Robert Schlögl, \\ and Thomas Lunkenbein*
}

Cite This: J. Phys. Chem. C 2020, 124, 23069-23077

Read Online

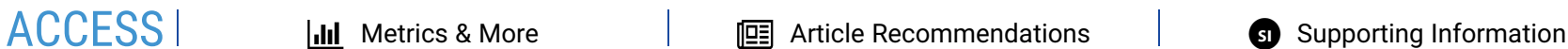

ABSTRACT: The local composition of heterogeneous catalysts can deviate from average values obtained by integral techniques. However, due to the difficulties in capturing nontranslational parts, they are often not determined. We have examined local alterations in the metal cation distribution of open-structured orthorhombic $(\mathrm{Mo}, \mathrm{V}) \mathrm{O}_{x}$ by scanning transmission electron microscopy coupled to electron energy loss spectroscopy (STEM-EELS). A HyperSpy-based routine was adapted and implemented that allows for automated EELS analysis. This analysis revealed compositional variations that reflect differences among the bulk, termination layers, and different surface facets. For instance, the lateral surface is preferentially enriched in molybdenum compared to the bulk, while V-rich surfaces can also be detected for the basal plane. These compositional inhomogeneities can modulate the surface reactivity on the atomic scale. The knowledge of local compositional

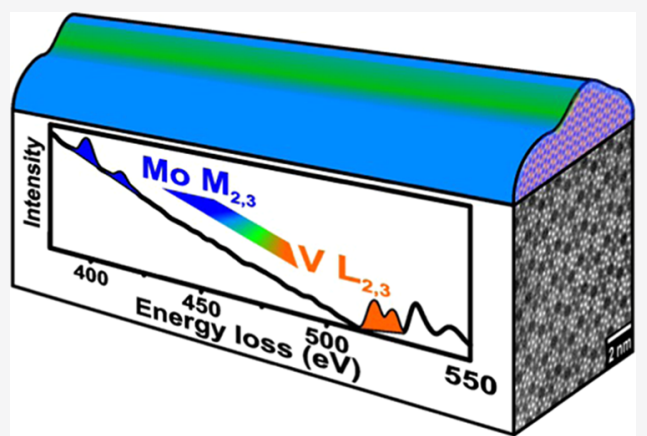
gradients is of general importance for any material as they alter its local chemistry and, thus, its physicochemical properties. Furthermore, such knowledge allows establishing novel composition-activity correlations, which could provide a deeper understanding of the origin of the function of materials.

\section{INTRODUCTION}

The composition of functional materials crucially influences their performances. Important examples include $\mathrm{p}$ - and n-type doping in semiconducting physics or the influencing factor of promoters for conversion or selectivity in heterogeneous catalysis. ${ }^{1}$ To fully understand the performance of any material, knowledge of the average composition is not sufficient. Compositional deviations occur locally and are generally overseen by common averaging analytical techniques. Experiments dedicated to capture the local composition are considered difficult and are, due to complexity, often not conducted. However, information on the local composition is an essential addition onto our current view on the origin of the function of materials.

Several techniques can be used to derive an integral estimate of the chemical composition of solids. ${ }^{2}$ For example, X-ray and ultraviolet photoelectron spectroscopy (XPS and UPS) allow determining the near-surface composition, while chemical analyses such as inductively coupled plasma optical emission spectrometry (ICP-OES) and X-ray fluorescence (XRF) provide compositional information of the entire sample. ${ }^{3}$ Other techniques, such as X-ray diffraction (XRD) analysis, rely on an average intensity distribution obtained by analyzing the coherent scattering parts of the sample. ${ }^{3}$ The examination of incoherent scattering parts of the sample, such as defects and surfaces, requires specific analysis of XRD peak profiles, which results in integral information. ${ }^{4}$ Local information can be obtained by (scanning) transmission electron microscopy ((S)TEM).$^{5-7}$ In particular, local structural analyses conducted by modern aberration-corrected STEM instruments allow identifying the differences between the bulk and surface structure of crystalline and amorphous materials. ${ }^{8-12}$ However, for complex, multielement materials, any detailed and direct conclusion on local compositional irregularities by common Zcontrast imaging is limited due to mixed metal site occupation, local thickness variations, structural defects, or the presence of other ill-defined morphologies. The information depth of the recorded micrographs can be enhanced by coupling STEM with analytical techniques, such as energy-dispersive X-ray spectroscopy (EDS) or electron energy loss spectroscopy (EELS). ${ }^{13-16}$ These techniques are sensitive to the elemental composition, and EELS can also allow tracking the electronic states of the involved elements. $^{17-19}$

Here, we consider as an example an open-structured oxide, formed with molybdenum and vanadium, and we decode local

Received: May 27, 2020

Revised: September 11, 2020

Published: October 13, 2020

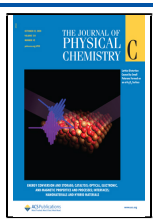


compositional variations by combining STEM and EELS analyses. Ternary $(\mathrm{Mo}, \mathrm{V}) \mathrm{O}_{x}$ crystallizes in an orthorhombic crystal structure. ${ }^{20-23}$ Oxides of this family combine structural complexity with catalytic activity ${ }^{20,23,24}$ and have been the subject of a multitude of structural STEM studies, also including the examination of defects. ${ }^{5-7,25-28}$ In brief, orthorhombic bimetallic $(\mathrm{Mo}, \mathrm{V}) \mathrm{O}_{x}$ is composed of pentagonal building blocks, which are connected by corner-sharing metal-oxygen octahedra so that structural hexagonal and heptagonal channels are formed (Figure 1). ${ }^{20,23}$ Figure 1 serves as a structural and

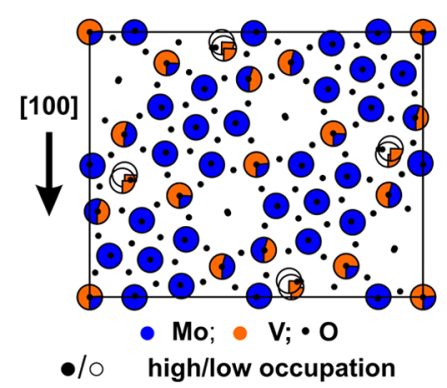

Figure 1. Unit cell representation of orthorhombic $(\mathrm{Mo}, \mathrm{V}) \mathrm{O}_{x}$ viewed along [001] derived from ref 23 .

compositional example that relies on averaged coherent scattering data. In this figure, the unit cell representation highlights local inhomogeneities related to the structure factor and reflects the average atom density distribution within the unit cell. $^{23}$ Our STEM-EELS analysis qualitatively compares changes in the composition of surface and bulk and will show that the molybdenum and vanadium enrichment can fluctuate between different parts of the sample. The lateral surface of the rodlike $(\mathrm{Mo}, \mathrm{V}) \mathrm{O}_{x}$ crystals is found to be preferentially enriched by molybdenum, whereas the basal plane is enriched in vanadium. The automated and reproducible extraction of individual data points from the STEM-EELS measurements was achieved by the implementation and adaption of a HyperSpy ${ }^{29}$-based routine. This routine can be prospectively applied to the analysis of EEL spectra of different elements and can be extended to the evaluation of big EELS data stacks. The applied HyperSpy-based routine is provided in the Supporting Information. The investigation may not provide a statistical analysis but provides important local information concerning the composition of the surface, which can be used by theory to establish more realistic models and to predict the performance of different surface terminations.

\section{METHODS}

Synthesis. Orthorhombic $(\mathrm{Mo}, \mathrm{V}) \mathrm{O}_{x}$ mixed oxide (FHI internal ID: 20000) was prepared via hydrothermal synthesis in an autoclave (Premex Reactor AG, Lengnau, Switzerland) built from corrosion-resistant Hastelloy C-22 (2.4602). The detailed synthesis procedure is presented elsewhere. ${ }^{7}$ In brief, a mixture of $9.18 \mathrm{~g}$ of $\left(\mathrm{NH}_{4}\right)_{6} \mathrm{Mo}_{7} \mathrm{O}_{24} \cdot 4 \mathrm{H}_{2} \mathrm{O}$ (Merck, $52 \mathrm{mmol} \mathrm{Mo}$ ) dissolved in $230 \mathrm{~g}$ of water (Milli-Q, Merck) and $3.30 \mathrm{~g}$ of $\mathrm{VOSO}_{4}$ (Acros Organics, $12.9 \mathrm{mmol} \mathrm{V}$ ) dissolved in $30 \mathrm{~g}$ of water was poured into the autoclave at room temperature (RT). Residual air was replaced by flushing with $\mathrm{N}_{2}$. Subsequently, the vessel was heated at a rate of $1{ }^{\circ} \mathrm{C} / \mathrm{min}$ to $200^{\circ} \mathrm{C}$ and dwelled for $17 \mathrm{~h}$. The reaction mixture was stirred at $100 \mathrm{rpm}$ during the entire experiment. After cooling to RT, a black solid was isolated by filtration (pore 5 glass frit), washed twice with distilled water, and dried at $80^{\circ} \mathrm{C}$ for $16 \mathrm{~h}$. To remove amorphous components in the hydrothermal product, $1 \mathrm{~g}$ of the powder was washed with $25 \mathrm{~mL}$ of oxalic acid solution $(0.25 \mathrm{M}$, Acros Organics $)$ at $60{ }^{\circ} \mathrm{C}$ for $30 \mathrm{~min}$ under stirring. The solid was centrifuged $(5000 \mathrm{rpm}$, $30 \mathrm{~min}$ ), washed twice with $25 \mathrm{~mL}$ of water, and dried at $80{ }^{\circ} \mathrm{C}$ overnight. Finally, a thermal treatment was performed in 100 $\mathrm{mL} / \mathrm{min}$ Ar flow at $400^{\circ} \mathrm{C}$ (heating rate $10^{\circ} \mathrm{C} / \mathrm{min}$ ) for $2 \mathrm{~h}$ in a rotary tube furnace (Xerion, Freiberg, Germany).

$X R D$. The X-ray diffraction measurements were performed in Bragg-Brentano geometry on a Bruker AXS D8 Advance II theta/theta diffractometer using Ni-filtered $\mathrm{Cu} \mathrm{K} \alpha$ radiation and a position-sensitive energy-dispersive LynxEye silicon strip detector. The sample powder was filled into the recess of a cup-shaped sample holder, the surface of the powder bed being on a level with the sample holder edge (front loading).

XPS. XPS spectra were recorded from sample pellets at RT using non-monochromatized $\mathrm{Al} \mathrm{K} \alpha(1486.6 \mathrm{eV})$ excitation and a hemispherical analyzer (Phoibos 150, SPECS). The binding energy scale was calibrated by the standard $\mathrm{Au} 4 \mathrm{f}_{7 / 2}$ and $\mathrm{Cu} 2 \mathrm{p}_{3 / 2}$ procedure. To calculate the elemental composition, theoretical cross sections from Yeh and Lindau were used. ${ }^{30}$

Chemical Analysis. The quantitative analysis of Mo and V was done by applying a closed-vessel high-pressure microwaveassisted digestion procedure (ETHOS plus 2, MLS). Three accurately weighed samples $(8 \mathrm{mg}$ ) were decomposed in $3 \mathrm{~mL}$ of aqua regia at a temperature of $160^{\circ} \mathrm{C}$ for $20 \mathrm{~min}$. The solution was transferred into volumetric flasks $(100 \mathrm{~mL})$ and filled up with ultrapure water. The solutions were measured against a matrix-matched calibration series with inductively coupled plasma optical emission spectrometry (Agilent 5100 SVDV ICP-OES.).

(S)TEM. TEM investigations were performed at $200 \mathrm{kV}$ using a JEOL JEM-ARM200 CF microscope equipped with CEOS CESCOR and CEOS CETCOR hexapole aberration correctors for probe and image-forming lenses, respectively, and a cold field emission gun (CFEG). Prior to the measurements, the powdered material was drop-deposited on a silica-coated gold TEM grid or carbon-coated copper TEM grid. Some particles were oriented along the crystallographic $c$-axis in the TEM mode. STEM-EELS images were recorded with a GATAN highangle annular dark-field (HAADF) detector. The STEM image that highlights the particle, which is presented in the energyfiltered TEM (EFTEM) map in Figure S11, was recorded with a JEOL ADF detector. The current was set between $1.8 \times 10^{7}$ and $8.6 \times 10^{7} \mathrm{e} / \mathrm{s}$ and controlled by a Gatan 643 TEM holder equipped with a Faraday cup.

EFTEM. EFTEM data were collected by a Gatan Imaging Filter (Quantum) CCD camera applying a monochromator slit of $10 \mathrm{eV}$ width for zero-loss collection. Thickness maps were obtained from the ratio of unfiltered and zero-loss maps and further color enhanced for clarity.

EELS. EEL spectra were collected using a Gatan Imaging Filter (Quantum), a dispersion of $0.25 \mathrm{eV}$, pixel times lower than or equal to $0.5 \mathrm{~s}$ for the core-loss regions and lower than or equal to $2.0 \times 10^{-3} \mathrm{~s}$ for the zero loss, emission currents below or equal to $8 \mu \mathrm{A}$, pixel sizes larger than or equal to $3 \AA$, a collection semiangle of $88 \mathrm{mrad}$, and a convergence semiangle of $22 \mathrm{mrad}$. Low- and high-loss spectra were recorded simultaneously using dual-EELS mode in the Gatan DigitalMicrograph software. Drift correction was applied after each 10 pixels. HAADF-STEM images of the examined area were collected before and after each scan, and drift correction area was further used as a reference to confirm the absence of beam damage. 
EELS Evaluation. The evaluation of the vanadium content in oxides is not trivial as the major vanadium $\mathrm{L}_{2,3^{-}}$and oxygen $\mathrm{K}$ edges partially overlap. As has been reported in the literature, ${ }^{31}$ the general approach for the determination of the metal content in related oxides involves the intensities of the $\mathrm{V} \mathrm{L}_{2,3}$-edges. However, if oxygen or additional oxide species as in mixed metal oxides are present, these intensities are difficult to estimate precisely. A more robust approach for the extraction of the $\mathrm{V}$ content requires the integration of the $\mathrm{V} \mathrm{L}_{2,3}$-edges prior to the onset of the $\mathrm{O}$ K-edge. ${ }^{32}$ For both $\mathrm{Mo}^{33}$ and $\mathrm{V}^{32,34-37}$ oxides, a comparably late onset of the $\mathrm{O}$ K-edge $(>528 \mathrm{eV})$ is observed. To estimate the influence of the possible overlap of $\mathrm{V} \mathrm{L}_{2,3}$ and $\mathrm{O}$ $\mathrm{K}$-edges on the integrated intensity distribution, the corresponding peaks were fitted by multiple Gaussians, and the overlap between these fits was used to indicate the precision of the evaluation. It is reflected in the error bars of the measurements.

For Mo, it is possible to use several different M-edges for quantification. ${ }^{33,38}$ However, the major Mo $\mathrm{M}_{4,5}$-edges demonstrate a delayed onset, ${ }^{39}$ and more importantly, these edges overlap with the C K-edge, which can lead to overestimation of the Mo content due to carbon contamination or the presence of the carbon support on the TEM grid. A proper background subtraction can be applied for the $\mathrm{M}_{2,3}$-edges, which would allow for excluding any overlapping influence of the C K-edge. ${ }^{33}$

To minimize beam artifacts, STEM-EELS line scans were recorded with intermediate resolution, which renders it difficult to determine the exact position. Calibration of the scan position was obtained by taking the different thicknesses/atomic densities of the open structure of orthorhombic $(\mathrm{Mo}, \mathrm{V}) \mathrm{O}_{x}$ into account, which results in different inelastic scattering events. This factor is reflected by the so-called relative thickness value evaluated from the low-loss spectra measured at each point of the line scan. The relative thickness $t / \lambda$ is conventionally described as

$$
t / \lambda=\log \left(I_{0} / I\right)
$$

where $t$ is the absolute sample thickness, $\lambda$ corresponds to the inelastic mean free path, which depends on the elemental composition, $I_{0}$ is the intensity originating from the zero-loss peak, and $I$ denotes the intensity of the entire spectrum. ${ }^{15}$ In contrast to the absolute thickness $t$, a relative thickness $t / \lambda$ can be precisely calculated without the exact knowledge of the composition and is given in units of inelastic mean free path (IMFP). ${ }^{15}$ Below relative thicknesses of 0.3 , multiple scattering can be neglected. ${ }^{40}$

To obtain a satisfactory and reproducible evaluation, the following procedure was performed (see Supporting Information for further details)

(1) The high- and low-loss spectrum images were simultaneously aligned using the zero-loss peak position as a reference.

(2) The background was subtracted using an inverse power law function fitting in the range prior to the onsets of Mo $\mathrm{M}_{4,5^{-}}$and $\mathrm{C}$ K-edges $(212-230 \mathrm{eV})$.

(3) If necessary $(t / \lambda>0.3)$, multiple scattering was removed using the Fourier-ratio method.

(4) The background prior to Mo $\mathrm{M}_{2,3}$-edges was subtracted using two power law functions with the same power but different amplitudes for ranges prior to $\mathrm{Mo}_{2,3^{-}}$and $\mathrm{V}$ $\mathrm{L}_{2,3}$-edges.

(5) A two-step Heaviside function was subtracted from the Mo $\mathrm{M}_{2,3}$-edge range. ${ }^{33}$
(6) If the signal-to-noise ratio in the $\mathrm{Mo} \mathrm{M}_{2,3}$-edges was higher than 2 , then the evaluation of the spectrum was performed. The evaluation included

(a) Integration of the spectrum in the range of the $\mathrm{Mo} \mathrm{M}_{2,3^{-}}$ edges $(390-425 \mathrm{eV})$.

(b) Fitting of the spectrum in the range of the $\mathrm{V} \mathrm{L}_{2,3}$ - and $\mathrm{O} \mathrm{K}$ edges using four Gaussian functions, where the first two ones describe the $\mathrm{VL}_{2,3}$-edges and the last two ones the $\mathrm{O}$ K-edge.

(c) The areas of the $\mathrm{VL}_{2,3}$-edges were calculated as the sum of the areas of the two corresponding Gaussian peaks.

(d) The overlap between $\mathrm{V}$ and $\mathrm{O}$ fitting functions is used to estimate the precision of the calculations.

The full procedure is demonstrated in Figure S1, and the adapted and applied HyperSpy ${ }^{29}$-based documentation is presented in the Supporting Information.

Atomic ratios of the metals in the sample $N_{\mathrm{Mo}} / N_{\mathrm{V}}$ can be correlated with intensities of the characteristic $\mathrm{Mo} \mathrm{M}_{2,3}$ and $\mathrm{V}$ $\mathrm{L}_{2,3}$-edges $\left(I_{\mathrm{Mo}}\right.$ and $\left.I_{\mathrm{V}}\right)$ using inelastic scattering cross sections $\sigma_{\mathrm{Mo}}$ and $\sigma_{\mathrm{V}}^{41}$

$$
\frac{N_{\mathrm{Mo}}}{N_{\mathrm{V}}}=\frac{I_{\mathrm{Mo}}}{I_{\mathrm{V}}} \frac{\sigma_{\mathrm{V}}}{\sigma_{\mathrm{Mo}}}
$$

Inelastic scattering cross sections describe the probability of the electron beam to excite electrons in the atomic shell of an element. ${ }^{41}$ In EELS experiments, the inelastic scattering cross sections depend not only on the energy of the specific atomic shell but also on the conditions of the spectrum acquisition. These include the collection (semi-) angle $\beta$ and the integration windows $\Delta_{\mathrm{Mo}, \mathrm{V}}$, in which the intensities of the target areas of the spectra are determined. ${ }^{41}$ For EEL spectra that were collected under identical conditions, the ratio $\sigma_{\mathrm{V}} / \sigma_{\mathrm{Mo}}$ can be treated as a proportionality constant (Figure S2). Observed variations in the integrated intensity ratio $I_{\mathrm{Mo}} / I_{\mathrm{V}}$ of the respective core-loss edges can, thus, be used to conclude qualitatively on trends in the local $\mathrm{Mo} / \mathrm{V}$ ratio of individual particles.

\section{RESULTS}

STEM-EELS Analysis. The selected orthorhombic oxide $\left(\mathrm{MoV}_{0.42}\right) \mathrm{O}_{x}$ (elemental composition according to ICP-OES) is phase-pure according to Rietveld-refined XRD data (Figure S3). A statistical structural evaluation of an identical material, which includes details on its rodlike character and a catalog of defects, is presented elsewhere.

Complementary to XRD, STEM-EELS measurements allow for combining experiments that tackle the local geometry with the extraction of electronic and compositional information on solids beyond translational symmetry from micrometer down to atomic scales. ${ }^{17,18}$ This can be achieved by simultaneously collecting EEL spectra of the low- and core-loss regions for each pixel of the corresponding STEM image (Figure 2a). The lowloss spectrum provides information on the relative thickness of the sample and is needed to remove multiple scattering contributions from the corresponding core-loss spectra of thicker samples. Furthermore, core-loss spectra are elementspecific and features of their characteristic edges can be used as an electronic fingerprint of an element, for instance, of molybdenum and vanadium (Figure $2 \mathrm{~b}$ ).

Orthorhombic $(\mathrm{Mo}, \mathrm{V}) \mathrm{O}_{x}$ is sensitive to the electron beam. Thus, we have adjusted the electron beam dose such that, throughout this study, the measurement of beam-induced artifacts could be avoided (Figures S4 and S5). However, at 

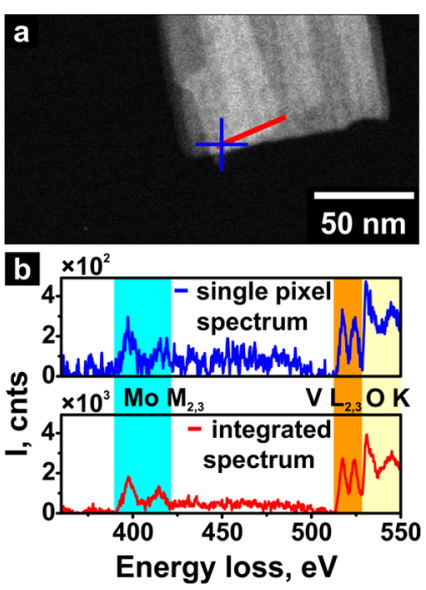

Figure 2. STEM-EELS measurements of orthorhombic $(\mathrm{Mo}, \mathrm{V}) \mathrm{O}_{x^{*}}$ (a) High-angle annular dark-field (HAADF)-STEM image of one orthorhombic $(\mathrm{Mo}, \mathrm{V}) \mathrm{O}_{x}$ rod. Blue cross: individual pixel that corresponds to the top spectrum in $\mathrm{b}$. Red line: position of the line scan of the EELS spectrum presented in the bottom part of b. (b) Coreloss spectra of an individual pixel (top) and integrated along the whole line scan (bottom) after background subtraction prior to the $\mathrm{Mo}_{2,3^{-}}$ edges and removal of multiple scattering; colored regions mark different edges.

sufficiently low beam doses, the individual spectrum of a single pixel suffers from a low signal-to-noise ratio (Figure $2 b$, top). Therefore, we accumulated measurements over a complete line scan to obtain an integrated EEL spectrum that can be used for further interpretation (Figure $2 \mathrm{~b}$, bottom). In addition, only line scans, which were recorded under identical conditions for the same nanorods, can be compared. Throughout the article, we used the integrated intensity ratio of $\mathrm{Mo} \mathrm{M}_{2,3}$ and $\mathrm{V} \mathrm{L}_{2,3}$-edges to describe changes of the local metal composition. Details on the evaluation of the EEL spectra and the applied HyperSpybased routine are given in the Methods section and Supporting Information.

STEM-EELS: Surface Enrichment. An example of such an analysis is given in Figure 3. The high-angle annular dark field (HAADF)-STEM image in Figure 3a displays the characteristic rodlike structure of orthorhombic $(\mathrm{Mo}, \mathrm{V}) \mathrm{O}_{x}$ with a preferential growth direction along the crystallographic $c$-axis. In addition, local contrast fluctuations along the nanorod hint at phases that have lower average $Z$ values compared to the surrounding, multiple open or closed pores of variable sizes. The mean HAADF-STEM intensity (Figure $3 \mathrm{~b}$ ) was evaluated along the yellow highlighted area of Figure $3 \mathrm{a}$ (starting in vacuum, across the surface to the bulk region; the signal is integrated along the perpendicular direction). The intensity scan (Figure $3 b$ ) shows several edges with different steepness values and the accurate positions of the STEM-EELS line scans. The HAADF-STEM intensity information presented in Figure $3 \mathrm{~b}$ can be correlated with a relative thickness value, which was obtained from the lowloss EEL spectra measured at different parts of the rod (Figures $3 \mathrm{c}$ and S6). Similar to the intensity distribution obtained from the HAADF-STEM image, the relative thickness derived from the low-loss EEL spectra indicates a thinner surface region compared to the bulk. In addition, throughout the article, we have chosen the relative thickness derived from the low-loss EEL spectra as a local anchor to derive the scan position precisely.

An evaluation of the STEM-EELS measurements is presented in Figure $3 \mathrm{c}$ and indicates a Mo enrichment along the thin lateral surface region of the particle compared to the thicker bulk part.

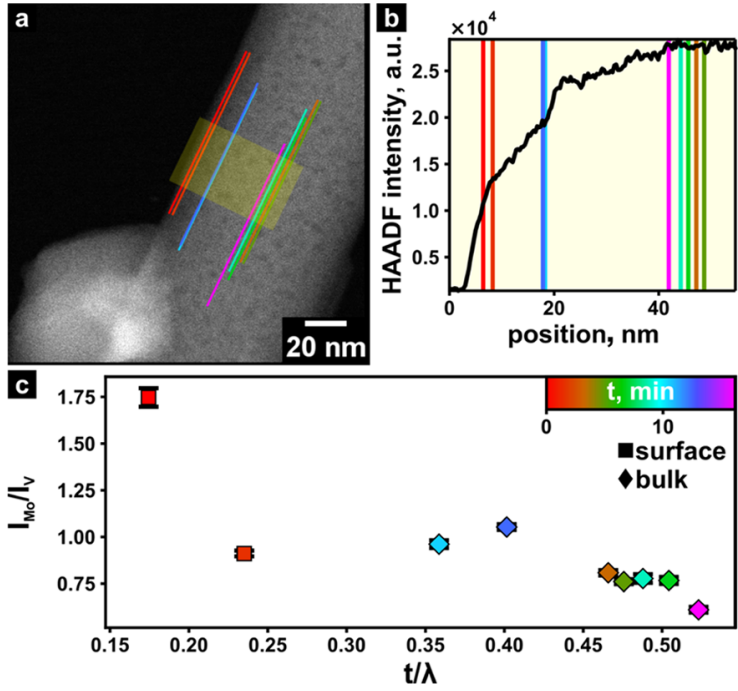

Figure 3. Mo/V integrated intensity ratio variations along the growth direction of orthorhombic $(\mathrm{Mo}, \mathrm{V}) \mathrm{O}_{x}$. (a) HAADF-STEM image of orthorhombic $(\mathrm{Mo}, \mathrm{V}) \mathrm{O}_{x}$. Yellow highlighted area: area used to extract the HAADF-STEM intensity profile; colored lines: exact scan positions. (b) Intensity profile of the HAADF-STEM image; colored lines: exact scan positions. (c) $I_{\mathrm{Mo}} / I_{\mathrm{V}}$ variation based on STEM-EELS measurements. The color code highlights the temporal order of STEM-EELS measurements.

The observation of a Mo-rich surface is consistent with surfaceaveraged XPS measurements that yield a nominal surface composition $\left(\mathrm{MoV}_{0.23}\right) \mathrm{O}_{x}$, which is a significantly higher Mo content than the corresponding nominal bulk composition $\left(\mathrm{MoV}_{0.42}\right) \mathrm{O}_{x}$ as determined by ICP-OES measurements. The finding of a Mo-rich surface along the growth direction was also confirmed for other $(\mathrm{Mo}, \mathrm{V}) \mathrm{O}_{x}$ rods (Figures S7). In addition, scan positions were arbitrarily selected with time to reduce a possible beam influence on vicinal positions of the analysis (Figures 3 and S7, color code). For STEM-EELS line scans repetitively recorded at different times and almost identical positions, the estimated Mo/V ratios show the same trend. The $\mathrm{Mo} / \mathrm{V}$ ratio can slightly vary around the expected value of the bulk composition and may highlight compositional anomalies that are present in the surface termination or in the bulk. A similar trend was obtained for the evaluation of the Mo $\mathrm{M}_{4,5^{-}}$ edges (Figure S8).

As has been shown in Figures 3 and S7, the dominant surface composition of $(\mathrm{Mo}, \mathrm{V}) \mathrm{O}_{x}$ along the lateral growth direction is richer in Mo compared to the bulk. However, the situation can change for the basal $(a, b)$ plane of orthorhombic $(\mathrm{Mo}, \mathrm{V}) \mathrm{O}_{x}$ (Figure 4). Figure $4 \mathrm{a}$ shows an additional HAADF-STEM image of this oxide. Besides the rodlike morphology and porosity, bright features can be observed that partially cover the rod. These prominent features are evident as oscillations that occur in the intensity profile of the bulk (Figure 4b). The highlighted scan positions indicate that the STEM-EELS line scans were conducted exclusively in local minima of such oscillations of the HAADF intensity to avoid any influence of the smaller particles on the determination of the composition. In addition, a sharp onset of the scan intensity can be observed at a position of around $10 \mathrm{~nm}$, indicating the beginning of the rod (Figure $4 \mathrm{~b}$ ). The corresponding integrated intensity ratio extracted from the surface of the $(a, b)$ plane and the bulk (Figure 4c) suggests a vanadium surface enrichment compared to the bulk. 


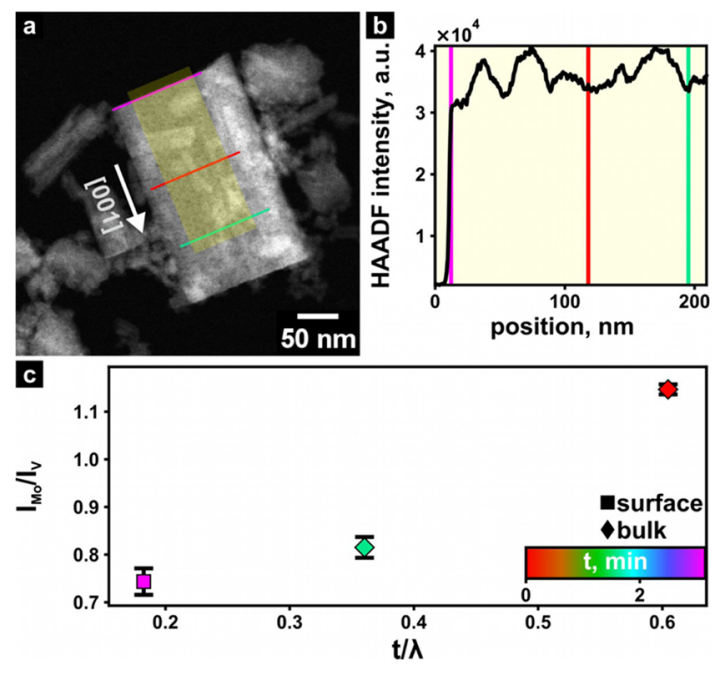

Figure 4. Compositional difference of the $(a, b)$ plane of orthorhombic $(\mathrm{Mo}, \mathrm{V}) \mathrm{O}_{x^{*}}$ (a) HAADF-STEM image of an orthorhombic $(\mathrm{Mo}, \mathrm{V}) \mathrm{O}_{x}$ rod; colored lines: exact scan positions. (b) Intensity profile of the HAADF-STEM image; colored lines: exact scan positions extracted from the yellow area in (a). (c) $I_{\mathrm{Mo}} / I_{\mathrm{V}}$ variation based on STEM-EELS measurements. The color code highlights the temporal order of STEMEELS measurements.

STEM-EELS: Surface Facets. The surface can be considered as a large defect in any material. Surfaces can be structurally decoupled from the bulk and their composition can vary depending on the exposed facet. ${ }^{7,42}$ This can lead to the occurrence of different surface reactivities within the same crystal. ${ }^{43}$ However, the determination of the composition of neighboring facets is quite difficult. An example of how minute local compositional differences at the surface of the oriented orthorhombic $(\mathrm{Mo}, \mathrm{V}) \mathrm{O}_{x}$ crystal can be is shown in Figure 5 where the HAADF-STEM image of a particle viewed along $[001]$ is given in Figure 5a. The HAADF-STEM image suggests

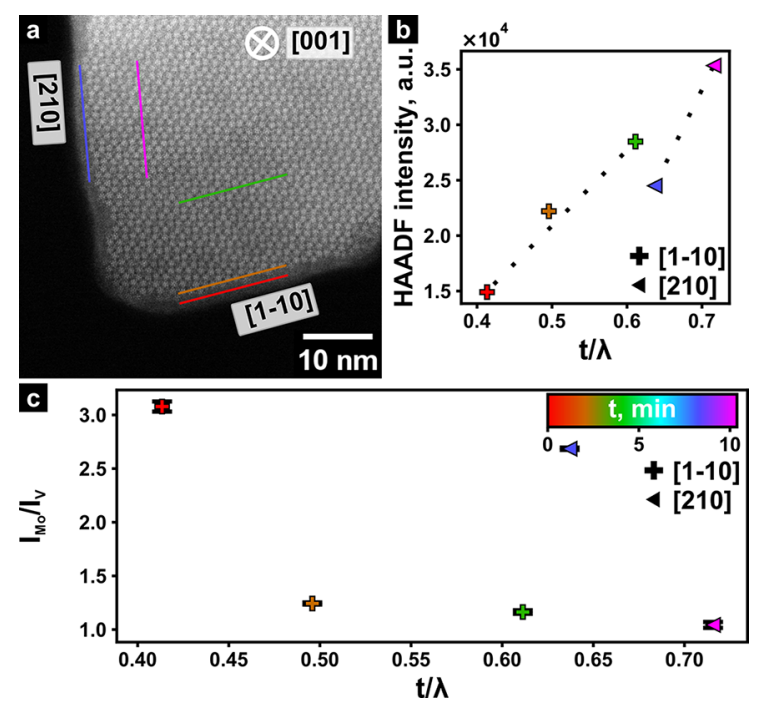

Figure 5. Local composition of different surface facets. (a) HAADFSTEM image of orthorhombic $(\mathrm{Mo}, \mathrm{V}) \mathrm{O}_{x}$ viewed along the crystallographic $c$-axis. (b) Mean HAADF intensity at the positions of the STEM-EELS line scans as a function of relative thickness. (c) $I_{\mathrm{Mo}} / I_{\mathrm{V}}$ variation as obtained from the STEM-EELS measurements. the presence of thin $(<1 \mathrm{~nm})$ amorphous surface layers that embed the crystalline bulk.

The same HAADF-STEM image further shows two different facets that grow in [210] and [1-10] directions, respectively. The linear correlation of HAADF-STEM line profiles and relative thickness presented in Figure $5 \mathrm{~b}$ demonstrates that for both facets the surface regions are thinner than the corresponding bulk regions. Figure $5 \mathrm{c}$ shows that these surfaces are terminated by Mo-rich layers compared to the bulk, while the Mo content of the $[1-10]$ terminated surface is slightly increased compared to the [210] terminated surface. An additional example is provided in Figure S9. Thus, the present STEM-EELS evaluation is capable of pinpointing surface compositional differences of vicinal facets. This knowledge is important to understand surface phenomena in functional materials.

Surface and Bulk Oxidation States. As has been shown above, bulk and surface are compositionally decoupled. Despite these local differences in the composition that were found by our detailed STEM-EELS line scan analyses, an additional trend has been observed and tackles the fine structure of the $\mathrm{O}$ K-edge (Figure 6). In Figure 6, EEL spectra of the $\mathrm{V}$ and $\mathrm{O}$ regions of the

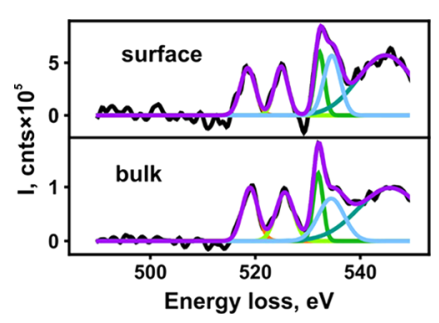

Figure 6. $\mathrm{VL}_{2,3}$ and $\mathrm{O}$ K-edges of the surface (top) and bulk (bottom) regions corresponding to the line scans along [210] presented in Figure 5 .

bulk (bottom) and surface (top) are shown. The high energy feature (blue Gaussian) of the $\mathrm{O} \mathrm{K}$ is substantially enhanced at the surface compared to the bulk (blue Gaussian). For comparison, EEL spectra of the surface and bulk of the Mo $\mathrm{M}_{2,3}$-edges are presented in Figure S10.

Discussion. A visual impression of how the local composition can influence an orthorhombic $(\mathrm{Mo}, \mathrm{V}) \mathrm{O}_{x}$ crystal is summarized in Figure 7 . It represents a $(\mathrm{Mo}, \mathrm{V}) \mathrm{O}_{x}$ nanorod at which a fragment was cut out along the crystallographic $c$-axis to gain insights into the bulk composition (green). The light green internal bulk corresponds to the average bulk composition. The top and bottom yellow surfaces characterize the V-enriched ( $a$, $b$ ) planes (Figure 4), which is in agreement with the occupation of the metal positions in the channels by vanadium as has been predicted before by single-crystal structure analysis. ${ }^{23}$ A cut perpendicular to the growth direction would expose these channel sites to the environment. The observed Mo-enriched surfaces (Figure 2) along the crystallographic $c$-axis are highlighted in blue, while the different shades of blue denote alterations in the Mo content of different surface terminations (Figure 5).

Existing reports demonstrate the possibility of resolving the composition of individual atomic columns of complex and dense packed oxides by STEM-EELS measurements. ${ }^{44,45}$ However, as opposed to their open-structured counterparts, these densely packed oxides are considered to be less sensitive to the electron beam. ${ }^{46}$ To the best of our knowledge, the local composition of 


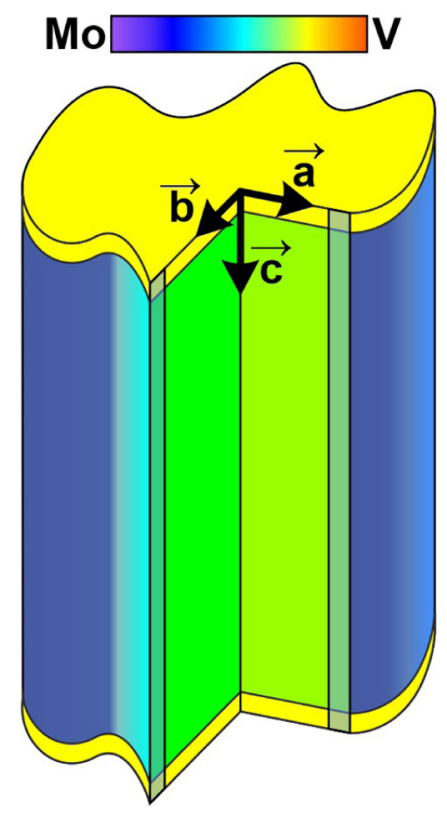

Figure 7. Local compositional distribution in an orthorhombic $(\mathrm{Mo}, \mathrm{V}) \mathrm{O}_{x}$ crystal. Black arrows: crystallographic axes; color bar: degree of enrichment.

individual parts (bulk and surface) of an open-structured molybdenum and vanadium mixed oxide has not been elucidated before. Although earlier reports, ${ }^{7,24,25,27,47}$ have discussed the presence of defects and local structures in molybdenum- and vanadium-containing oxides, conclusions on the local composition, in particular of the surface, are missing. Here, we have extended the previous knowledge about the geometric arrangement and metal site occupancies of complex $(\mathrm{Mo}, \mathrm{V}) \mathrm{O}_{x}^{7,11,20,21,23,47}$ to the local composition at the nanoscale, which can be best described by differences between the bulk and surface. Variations of the bulk composition are already known for multinary oxides and are related to differences in the metal site occupancy (Figure 1), which represents solutions of the average electron density. ${ }^{20,23,48}$ This average information can be obtained by diffraction experiments and is manifested in the electron density equation, ${ }^{49}$ while the local electron density is reflected by the relative thickness (Figures 3 and S11) and mass thickness contrast (Figure 3). The observed difference in the composition of the bulk and surface is also in line with observations made for other catalytically active oxides. ${ }^{42}$ The agreement with predictions from chemical analysis, X-ray spectroscopy, and single-crystal studies ${ }^{23}$ further demonstrates that the observed compositional inhomogeneities are not caused by the electron beam. Furthermore, the present study highlights that integral and local techniques are complementary and allow capturing macro- and nanoscopic compositional changes. It should be noted that only EEL spectra were evaluated for which repetitive measurements at identical positions did not show any influence of the electron beam (Figures S4 and S5). In addition, electron beam-induced selective diminishment of one of the elements at thin surface parts can be excluded as measurements along different surfaces show either Mo or $\mathrm{V}$ enrichment (Figures 3 and 4).

The observed local compositional gradients can influence the intrinsic catalytic properties of this functional material. For instance, it has been shown that vanadium and molybdenum compounds exhibit different chemical behaviors and reactiv- ities. ${ }^{50,51}$ In addition, the sudden change of adsorption energies for different Mo- or V-rich surfaces in the subnanometer regime could crucially influence conversion, selectivity, or oxygen activation. To which extent these local compositional gradients, summarized in Figure 7, can influence the reactivity and functionality of orthorhombic $(\mathrm{Mo}, \mathrm{V}) \mathrm{O}_{x}$ cannot be assessed experimentally and requires theoretical modeling. While the experimental assessment is not possible, the knowledge on compositional decoupling of the bulk, surface, and different facets is, thus, important as it may provoke new ideas for the design of novel and efficient selective oxidation catalysts.

However, not only the surface composition but also the local electronic state alters the catalytic performance. An interesting phenomenon is addressed in Figure 6. As has been previously shown, ${ }^{35}$ the $\mathrm{V} \mathrm{L}_{2}$ to $\mathrm{L}_{3}$ intensity ratio, their position on the energy scale, and the fine structure of the O K-edge can be indicative of the oxidation state of pure vanadium oxides. In $(\mathrm{Mo}, \mathrm{V}) \mathrm{O}_{x}$, the intensity ratio of the $\mathrm{V} \mathrm{L}_{2,3}$-edges may not only be influenced by the partial overlapping of the $\mathrm{V} \mathrm{L}_{2.3^{-}}$and $\mathrm{O} \mathrm{K}$ edges as it appears for pure $\mathrm{V}_{x} \mathrm{O}_{y}$ but also from oxygen contributions stemming from molybdenum oxides, ${ }^{52}$ which renders conclusion on the oxidation state solely derived from the behavior of the $\mathrm{V} \mathrm{L}_{2,3}$-edges impossible. It has been emphasized in previous studies that the high-energy feature of the oxygen fine structure (Figure 6, blue Gaussians) also correlates with the oxidation states in both pure vanadium and pure molybdenum oxides. $^{33,35}$ In both oxides, a partially reduced species is characterized by a high intensity of the high-energy feature of the $\mathrm{O} \mathrm{K}$ fine structure. A higher intensity of the high-energy feature of the fine structure of the $\mathrm{O}$ K-edge at the surface compared to the bulk has been observed in our study (Figure 6, blue Gaussian) and would suggest a slightly reduced surface. However, the energy loss near edge fine structure (ELNES) reflects the local unoccupied density of states and is, therefore, sensitive to the entire local chemical environment. ${ }^{41}$ Thus, the estimation of the local oxidation states in mixed oxides based on fingerprinting of the corresponding oxides is not always possible and difficult to disentangle from other effects. ${ }^{52}$ Hence, a precise oxidation state of the sample cannot be determined due to the limited signal-to-noise ratio, owing to the beam sensitivity of the sample. Although a change in the $\mathrm{O} \mathrm{K}$ fine structure can be identified, the $\mathrm{V}-\mathrm{L}_{2}$ to $\mathrm{V}-\mathrm{L}_{3}$ peak ratios (Figure 6) change only slightly, which could also be, as mentioned above, attributed to the oxygen contributions stemming from Mo. The Mo- $\mathrm{M}_{2}$ to Mo- $\mathrm{M}_{3}$ peak ratios (Figure S10) remain almost constant, independent of the surface and bulk area. Therefore, we exclude a possible variation of the oxidation state as the only source for the observed $\mathrm{Mo} / \mathrm{V}$ integrated peak intensity ratio change.

It has been recently addressed that the average surface oxidation state of $\mathrm{V}$ moieties in orthorhombic $(\mathrm{Mo}, \mathrm{V}) \mathrm{O}_{x}$ is 4.3 under operation conditions, which would indicate the presence of a partially reduced V surface. ${ }^{53,54}$ In addition, surface Mo species were found to occur mainly in their $\mathrm{d}^{0}$ states, while small fractions of reduced $\mathrm{d}^{1}$ species cannot be excluded. ${ }^{23}$

Controlling the crystal and compositional structures is challenging as both are closely related and depend on the interplay of several synthetic and postsynthetic parameters. Note that polymorphism in this kind of complex oxide is triggered by the $\mathrm{Mo} / \mathrm{V}$ ratio. In addition, if the solution parameters, such as temperature, $\mathrm{pH}$, and concentration, are not carefully adjusted, unwanted chemical processes may lead to the observed compositional gradients. Moreover, local composition differences between the surface and the bulk may be inherently 
related to the anisotropic crystal growth as they influence condensation rates. The preferential stacking along the $c$-axis relies on a particular distribution of Mo and V in which Mo is preferentially located in the pentagonal pillars that provide certain robustness to the growing crystal. The extension of the $(a, b)$ plane might then be accomplished by both Mo- and V-rich domains. Experimental evidence of the respective enrichment can be found in Figures 3-5. The choice of the reaction parameters and temperature treatment is crucial as it can influence the surface composition and, thus, the outcome of the reaction. It further controls the thermodynamic and kinetic properties of the desired functional solid.

Conclusions. In summary, the present study demonstrates that the local metal distribution in an open-structured oxide, as determined by combined STEM-EELS measurements, can differ more than $50 \%$ from that of the averaged crystal composition. A HyperSpy-based routine is provided that would prospectively allow for the automated evaluation of large EELS data stacks. Orthorhombic $(\mathrm{Mo}, \mathrm{V}) \mathrm{O}_{x}$ exhibits intrinsic compositional inhomogeneities at different scales, which can be found in the bulk and at the surface. The observations are consistent with results from single-crystal studies, chemical analysis, and XPS studies. These differences can be expressed by Mo- or V-surface enrichments compared to the bulk that can vary locally. Compositional variations occur even for vicinal surfaces. The impact of this finding on the catalytic activity and selectivity cannot be assessed experimentally. However, this information provides valuable surface models from which the reactivity can be calculated based on first principles, which may lead to new ideas in the design of prospective high-performance selective oxidation catalysts.

\section{ASSOCIATED CONTENT}

\section{SI Supporting Information}

The Supporting Information is available free of charge at https://pubs.acs.org/doi/10.1021/acs.jpcc.0c04777.

Analysis workflow; relationship between molar ratio and intensities; measured and refined XRD pattern; beam damage studies; scan positions; additional examples for the lateral surface; analysis of the $\mathrm{Mo} \mathrm{M}_{4,5}$-edge; additional examples of surface facet analysis; comparison of bulk and surface Mo $\mathrm{M}_{2,3}$-edges; relative thickness by EFTEM; and HyperSpy-based routine (PDF)

\section{AUTHOR INFORMATION}

\section{Corresponding Author}

Thomas Lunkenbein - Department of Inorganic Chemistry, Fritz-Haber-Institut der Max-Planck-Gesellschaft, 14195 Berlin, Germany; 이이이.org/0000-0002-8957-4216;

Email: lunkenbein@fhi-berlin.mpg.de

\section{Authors}

Liudmyla Masliuk - Department of Inorganic Chemistry, FritzHaber-Institut der Max-Planck-Gesellschaft, 14195 Berlin, Germany

Franz-Philipp Schmidt - Department of Inorganic Chemistry, Fritz-Haber-Institut der Max-Planck-Gesellschaft, 14195 Berlin, Germany; Department of Heterogeneous Reactions, Max Planck Institute for Chemical Energy Conversion, 45470 Mülheim an der Ruhr, Germany; (1) orcid.org/0000-0003-0125-2704

Walid Hetaba - Department of Inorganic Chemistry, FritzHaber-Institut der Max-Planck-Gesellschaft, 14195 Berlin,
Germany; Department of Heterogeneous Reactions, Max Planck Institute for Chemical Energy Conversion, 45470 Mülheim an der Ruhr, Germany

Milivoj Plodinec - Department of Inorganic Chemistry, FritzHaber-Institut der Max-Planck-Gesellschaft, 14195 Berlin, Germany

Gudrun Auffermann - Max Planck Institute for Chemical Physics of Solids, 01187 Dresden, Germany

Klaus Hermann - Department of Inorganic Chemistry, FritzHaber-Institut der Max-Planck-Gesellschaft, 14195 Berlin, Germany; 이이이.org/0000-0002-3861-3916

Detre Teschner - Department of Inorganic Chemistry, FritzHaber-Institut der Max-Planck-Gesellschaft, 14195 Berlin, Germany; Department of Heterogeneous Reactions, Max Planck Institute for Chemical Energy Conversion, 45470 Mülheim an der Ruhr, Germany

Frank Girgsdies - Department of Inorganic Chemistry, FritzHaber-Institut der Max-Planck-Gesellschaft, 14195 Berlin, Germany

Annette Trunschke - Department of Inorganic Chemistry, FritzHaber-Institut der Max-Planck-Gesellschaft, 14195 Berlin, Germany; 이이.orid.o000-0003-2869-0181

Robert Schlögl - Department of Inorganic Chemistry, FritzHaber-Institut der Max-Planck-Gesellschaft, 14195 Berlin, Germany; Department of Heterogeneous Reactions, Max Planck Institute for Chemical Energy Conversion, 45470 Mülheim an der Ruhr, Germany

Complete contact information is available at:

https://pubs.acs.org/10.1021/acs.jpcc.0c04777

\section{Notes}

The authors declare no competing financial interest.

\section{ACKNOWLEDGMENTS}

This work was conducted in the framework of the BasCat collaboration between BASF SE, TU Berlin, FHI, and the cluster of excellence "Unified Concepts in Catalysis" (UniCat www. unicat.tu-berlin.de). Johannes Noack is acknowledged for the synthesis of orthorhombic $(\mathrm{Mo}, \mathrm{V}) \mathrm{O}_{x}$ during his stay at $\mathrm{FHI}$.

\section{REFERENCES}

(1) Yu, P. Y.; Cardona, M. Electronic Properties of Defects. In Fundamentals of Semiconductors: Physics and Materials Properties, 4th ed., 2010; pp 159-202.

(2) Haber, J.; Block, J. H.; Delmon, B. Methods and Procedures for Catalyst Characterization. In Handbook of Heterogeneous Catalysis; Ertl, G.; Knözinger, H.; Schüth, F.; Weitkamp, J., Eds.; Wiley-VCH, 2008.

(3) Albers, P. W. Bulk Chemical Composition. In Handbook of Heterogeneous Catalysis, 2008.

(4) Kasatkin, I.; Kurr, P.; Kniep, B.; Trunschke, A.; Schlögl, R. Role of Lattice Strain and Defects in Copper Particles on the Activity of $\mathrm{Cu} /$ $\mathrm{ZnO} / \mathrm{Al}_{2} \mathrm{O}_{3}$ Catalysts for Methanol Synthesis. Angew. Chem., Int. Ed. 2007, 46, 7324-7327.

(5) Vogt, T.; Blom, D. A.; Jones, L.; Buttrey, D. J. ADF-STEM Imaging of Nascent Phases and Extended Disorder Within the Mo-VNb-Te-O Catalyst System. Top. Catal. 2016, 59, 1489-1495.

(6) Melzer, D.; Xu, P. H.; Hartmann, D.; Zhu, Y. Y.; Browning, N. D.; Sanchez-Sanchez, M.; Lercher, J. A. Atomic-Scale Determination of Active Facets on the MoVTeNb Oxide M1 Phase and Their Intrinsic Catalytic Activity for Ethane Oxidative Dehydrogenation. Angew. Chem., Int. Ed. 2016, 55, 8873-8877.

(7) Masliuk, L.; Heggen, M.; Noack, J.; Girgsdies, F.; Trunschke, A.; Hermann, K. E.; Willinger, M. G.; Schlögl, R.; Lunkenbein, T. 
Structural Complexity in Heterogeneous Catalysis: Cataloging Local Nanostructures. J. Phys. Chem. C 2017, 121, 24093-24103.

(8) MacLaren, I.; Ramasse, Q. M. Aberration-Corrected Scanning Transmission Electron Microscopy for Atomic-Resolution Studies of Functional Oxides. Int. Mater. Rev. 2014, 59, 115-131.

(9) Klyushin, A. Y.; Greiner, M. T.; Huang, X.; Lunkenbein, T.; Li, X.; Timpe, O.; Friedrich, M.; Havecker, M.; Knop-Gericke, A.; Schlögl, R. Is Nanostructuring Sufficient To Get Catalytically Active Au? ACS Catal. 2016, 6, 3372-3380.

(10) Li, X.; Lunkenbein, T.; Pfeifer, V.; Jastak, M.; Nielsen, P. K.; Girgsdies, F.; Knop-Gericke, A.; Rosowski, F.; Schlögl, R.; Trunschke, A. Selective Alkane Oxidation by Manganese Oxide: Site Isolation of $\mathrm{MnO}_{\mathrm{x}}$ Chains at the Surface of $\mathrm{MnWO}_{4}$ Nanorods. Angew. Chem., Int. Ed. 2016, 55, 4092-4096.

(11) Lunkenbein, T.; Girgsdies, F.; Wernbacher, A.; Noack, J.; Auffermann, G.; Yasuhara, A.; Klein-Hoffmann, A.; Ueda, W.; Eichelbaum, M.; Trunschke, A.; et al. Direct Imaging of Octahedral Distortion in a Complex Molybdenum Vanadium Mixed Oxide. Angew. Chem., Int. Ed. 2015, 54, 6828-6831.

(12) Dellby, N.; Krivanek, O. L.; Nellist, P. D.; Batson, P. E.; Lupini, A. R. Progress in aberration-corrected scanning transmission electron microscopy. J. Electron Microsc. 2001, 50, 177-185.

(13) Batson, P. E. Simultaneous STEM Imaging and Elecron-EnergyLoss Spectroscopy with Atomic-Column Sensitivity. Nature 1993, 366, 727-728.

(14) D’Alfonso, A. J.; Freitag, B.; Klenov, D.; Allen, L. J. Atomicresolution chemical mapping using energy-dispersive $\mathrm{X}$-ray spectroscopy. Phys. Rev. B 2010, 81, No. 100101(R).

(15) Egerton, R. F. TEM Applications of EELS. In Electron Energy-Loss Spectroscopy in the Electron Microscope; Egerton, R. F., Ed.; Springer US: Boston, MA, 2011; pp 293-397.

(16) Hitchcock, A. P.; Dynes, J. J.; Johansson, G.; Wang, J.; Botton, G. Comparison of NEXAFS Microscopy and TEM-EELS for Studies of Soft Matter. Micron 2008, 39, 311-319.

(17) Botton, G. A.; Lazar, S.; Dwyer, C. Elemental Mapping at the Atomic Scale Using Low Accelerating Voltages. Ultramicroscopy 2010, 110, 926-934.

(18) Lazar, S.; Shao, Y.; Gunawan, L.; Nechache, R.; Pignolet, A.; Botton, G. A. Imaging, Core-Loss, and Low-Loss Electron-Energy-Loss Spectroscopy Mapping in Aberration-Corrected STEM. Microsc. Microanal. 2010, 16, 416-424.

(19) Brydson, R. A Brief Review of Quantitative Aspects of Electron Energy Loss Spectroscopy and Imaging. Mater. Sci. Technol. 2000, 16, $1187-1198$.

(20) Ishikawa, S.; Kobayashi, D.; Konya, T.; Ohmura, S.; Murayama, T.; Yasuda, N.; Sadakane, M.; Ueda, W. Redox Treatment of Orthorhombic $\mathrm{Mo}_{29} \mathrm{~V}_{11} \mathrm{O}_{112}$ and Relationships between Crystal Structure, Microporosity and Catalytic Performance for Selective Oxidation of Ethane. J. Phys. Chem. C 2015, 119, 7195-7206.

(21) Konya, T.; Katou, T.; Murayama, T.; Ishikawa, S.; Sadakane, M.; Buttrey, D.; Ueda, W. An Orthorhombic $\mathrm{Mo}_{3} \mathrm{VO}_{\mathrm{x}}$ Catalyst most Active for Oxidative Dehydrogenation of Ethane Among Related Complex Metal Oxides. Catal. Sci. Technol. 2013, 3, 380-387.

(22) Katou, T.; Vitry, D.; Ueda, W. Structure Dependency of Mo-VO-based Complex Oxide Catalysts in the Oxidations of Hydrocarbons. Catal. Today 2004, 91-92, 237-240.

(23) Trunschke, A.; Noack, J.; Trojanov, S.; Girgsdies, F.; Lunkenbein, T.; Pfeifer, V.; Hävecker, M.; Kube, P.; Sprung, C.; Rosowski, F.; et al. The Impact of the Bulk Structure on Surface Dynamics of Complex Mo-V-based Oxide Catalysts. ACS Catal. 2017, 7, 3061-3071.

(24) Grasselli, R. K.; Buttrey, D. J.; DeSanto, P.; Burrington, J. D.; Lugmair, C. G.; Volpe, A. F.; Weingand, T. Active Centers in Mo-V-NbTe-O-x (Amm)oxidation Catalysts. Catal. Today 2004, 91-92, 251258.

(25) Wagner, J. B.; Timpe, O.; Hamid, F. A.; Trunschke, A.; Wild, U.; Su, D. S.; Widi, R. K.; Abd Hamid, S. B.; Schlögl, R. Surface texturing of Mo-V-Te-Nb-O-x selective oxidation catalysts. Top. Catal. 2006, 38, $51-58$.
(26) Zhu, Y. Y.; Sushko, P. V.; Melzer, D.; Jensen, E.; Kovarik, L.; Ophus, C.; Sanchez-Sanchez, M.; Lercher, J. A.; Browning, N. D. Formation of Oxygen Radical Sites on MoVNbTeOx by Cooperative Electron Redistribution. J. Am. Chem. Soc. 2017, 139, 12342-12345.

(27) Pyrz, W. D.; Blom, D. A.; Sadakane, M.; Kodato, K.; Ueda, W.; Vogt, T.; Buttrey, D. J. Atomic-level Imaging of Mo-V-O Complex Oxide Phase Intergrowth, Grain Boundaries, and Defects using HAADF-STEM. Proc. Natl. Acad. Sci. U.S.A. 2010, 107, 6152-6157.

(28) Aouine, M.; Epicier, T.; Millet, J. M. M. In Situ Environmental STEM Study of the MoVTe Oxide M1 Phase Catalysts for Ethane Oxidative Dehydrogenation. ACS Catal. 2016, 6, 4775-4781.

(29) de la Peña, F.; Prestat, E.; Fauske, V. T.; Burdet, P.; Petras, J.; Nord, M.; Ostasevicius, T.; MacArthur, K.; Sarahan, M.; Johnstone, D. N.. et al.HyperSpy, v1.5.2. http://doi.org/10.5281/zenodo.3396791 2019.

(30) Yeh, J. J.; Lindau, I. Atomic subshell photoionization cross sections and asymmetry parameters: $1 \leq \mathrm{Z} \leq 103$. At. Data Nucl. Data Tables 1985, 32, 1-155.

(31) Pearson, D. H.; Ahn, C. C.; Fultz, B. White Lines and d-Electron Occupancies for the $3 \mathrm{~d}$ and $4 \mathrm{~d}$ Transition-Metals. Phys. Rev. B 1993, 47, $8471-8478$

(32) Ek, M.; Ramasse, Q. M.; Arnarson, L.; Georg Moses, P.; Helveg, S. Visualizing Atomic-scale Redox Dynamics in Vanadium Oxide-based Catalysts. Nat. Commun. 2017, 8, No. 305.

(33) Lajaunie, L.; Boucher, F.; Dessapt, R.; Moreau, P. Quantitative Use of Electron Energy-Loss Spectroscopy Mo- $\mathrm{M}_{2,3}$ Edges for the Study of Molybdenum Oxides. Ultramicroscopy 2015, 149, 1-8.

(34) Su, D. S.; Hebert, C.; Willinger, M.; Schlögl, R. Anisotropy and Collection Angle Dependence of the Oxygen K ELNES in $\mathrm{V}_{2} \mathrm{O}_{5}$ : a Band-structure Calculation Study. Micron 2003, 34, 227-233.

(35) Hébert, C.; Willinger, M.; Su, D. S.; Pongratz, P.; Schattschneider, P.; Schlögl, R. Oxygen K-edge in Vanadium Oxides: Simulations and Experiments. Eur. Phys. J. B 2002, 28, 407-414.

(36) Su, D. S.; Wieske, M.; Beckmann, E.; Blume, A.; Mestl, G.; Schlögl, R. Electron Beam Induced Reduction of $\mathrm{V}_{2} \mathrm{O}_{5}$ Studied by Analytical Electron Microscopy. Catal. Lett. 2001, 75, 81-86.

(37) Li, J.; Gauntt, B. D.; Kulik, J.; Dickey, E. C. Stoichiometry of Nanocrystalline $\mathrm{VO}_{\mathrm{x}}$ Thin Films Determined by Electron Energy Loss Spectroscopy. Microsc. Microanal. 2009, 15, 1004-1005.

(38) Hofer, F. EELS Quantification of M-Edges by Using Oxidic Standards. Ultramicroscopy 1987, 21, 63-67.

(39) Chen, J.; Wei, Q. Phase Transformation of Molybdenum Trioxide to Molybdenum Dioxide: An in-situ Transmission Electron Microscopy Investigation. Int. J. Appl. Ceram. Technol. 2017, 14, 10201025.

(40) Egerton, R. F. Physics of Electron Scattering. In Electron EnergyLoss Spectroscopy in the Electron Microscope; Egerton, R. F., Ed.; Springer US: Boston, MA, 2011; pp 111-229.

(41) Egerton, R. F., Quantitative Analysis of Energy-Loss Data. In Electron Energy-Loss Spectroscopy in the Electron Microscope, Egerton, R. F., Ed. Springer US: Boston, MA, 2011; pp 231-291.

(42) Li, X.; Teschner, D.; Streibel, V.; Lunkenbein, T.; Masliuk, L.; Fu, T.; Wang, Y. Q.; Jones, T.; Seitz, F.; Girgsdies, F.; et al. How to Control Selectivity in Alkane Oxidation? Chem. Sci. 2019, 10, 2429-2443.

(43) Grasselli, R. K. Fundamental Principles of Selective Heterogeneous Oxidation Catalysis. Top. Catal. 2002, 21, 79-88.

(44) Saito, M.; Kimoto, K.; Nagai, T.; Fukushima, S.; Akahoshi, D.; Kuwahara, H.; Matsui, Y.; Ishizuka, K. Local Crystal Structure Analysis with 10-pm Accuracy Using Scanning Transmission Electron Microscopy. J. Electron Microsc. 2009, 58, 131-136.

(45) Wu, H. J.; Zhao, X. X.; Song, D. S.; Tian, F.; Wang, J.; Loh, K. P.; Pennycook, S. J. Progress and Prospects of Aberration-corrected STEM for Functional Materials. Ultramicroscopy 2018, 194, 182-192.

(46) Jiang, N. Electron Beam Damage in Oxides: a Review. Rep. Prog. Phys. 2016, 79, No. 016501.

(47) Pyrz, W. D.; Blom, D. A.; Sadakane, M.; Kodato, K.; Ueda, W.; Vogt, T.; Buttrey, D. J. Atomic-Scale Investigation of Two-Component MoVO Complex Oxide Catalysts Using Aberration-Corrected High- 
Angle Annular Dark-Field Imaging. Chem. Mater. 2010, 22, 20332040.

(48) DeSanto, P.; Buttrey, D. J.; Grasselli, R. K.; Lugmair, C. G.; Volpe, A. F.; Toby, B. H.; Vogt, T. Structural Characterization of the Orthorhombic Phase M1 in MoVNbTeO Propane Ammoxidation Catalyst. Top. Catal. 2003, 23, 23-38.

(49) Coppens, P. The Structure Factor. In International Tables for Crystallography, Vol. B:Reciprocal Space, 3rd ed., Springer: Dordrecht, 2008; pp 10-23.

(50) Sebenik, R. F.; Burkin, A. R.; Dorfler, R. R.; Laferty, J. M.; Leichtfried, G.; Meyer-Grünow, H.; Mitchell, P.; Vukasovich, M. S.; Church, D. A.; van Riper, G. G. et al. Ullmannn's Encyclopedia of Industrial Chemistry, Molybdenum and Molybdenum Compounds; WileyVCH: Weinheim, 2000.

(51) Bauer, G.; Güther, V.; Hess, H.; Otto, A.; Roidl, O.; Roller, H.; Sattelberger, S.; Köther-Becker, S.; Beyer, T. Ullmann's Encyclopedia of Industrial Chemistry, Vanadium and Vanadium Compounds; WileyVCH: Weinheim, 2017.

(52) Wagner, J. B.; Su, D. S.; Schunk, S. A.; Hibst, H.; Petzoldt, J.; Schlögl, R. Structural Characterization of High-performance Catalysts for Partial Oxidation-the High-resolution and Analytical Electron Microscopy Approach. J. Catal. 2004, 224, 28-35.

(53) Wernbacher, A. M.; Eichelbaum, M.; Risse, T.; Cap, S.; Trunschke, A.; Schlögl, R. Operando Electrical Conductivity and Complex Permittivity Study on Vanadia Oxidation Catalysts. J. Phys. Chem. C 2019, 123, 8005-8017.

(54) Wernbacher, A. M.; Kube, P.; Havecker, M.; Schlögl, R.; Trunschke, A. Electronic and Dielectric Properties of MoV-Oxide (M1 Phase) under Alkane Oxidation Conditions. J. Phys. Chem. C 2019, 123, 13269-13282. 